\title{
\%
}

\section{EL SECTOR BANCARIO CHINO COMO CATALIZADOR DE LA RECUPERACIÓN ECONÓMICA TRAS LA COVID-19}

En menos de un año, China ha logrado contener la pandemia de la COVID-19 y retomar progresivamente la actividad económica a través de un ambicioso plan de estímulo fiscal, monetario y sectorial. Para ejecutar estas medidas de estímulo las autoridades han recurrido al sistema bancario chino como principal mecanismo de transmisión, incrementando los riesgos del propio sistema bancario, que podrían verse agravados en 2021. Las autoridades chinas, conscientes de este peligro, están gestionando selectivamente estos riesgos para intentar consolidar la recuperación económica iniciada en 2020.

Palabras Clave: banca en la sombra, China, sistema financiero, crisis económica, coronavirus, política económica.

Códigos JEL: G21, G23, G28, H12, N25, O53.

\section{Introducción}

En enero de 2020, China fue el primer país en sufrir la pandemia de la COVID-19 y, gracias a la aplicación de estrictas medidas de contención, ha sido el primero en vencerla de manera efectiva. Al mismo tiempo, a partir del mes de febrero, las autoridades comenzaron a aprobar numerosas medidas de estímulo de índole fiscal, monetario y sectorial para intentar contrarrestar el profundo impacto negativo causado por el brote de COVID-19.

\footnotetext{
* Técnico Comercial y Economista del Estado.

** Diplomada Comercial del Estado.

Las opiniones expresadas solo comprometen a sus autores.

Versión de enero de 2021.

DOI: https:/doi.org/10.32796/bice.2021.3132.7151
}

Para ejecutar estas medidas de estímulo, las autoridades chinas han recurrido al sistema bancario como principal mecanismo de transmisión, autorizando la relajación de algunas normas de supervisión y control para permitir a las entidades bancarias aumentar la financiación y garantizar la liquidez de la economía china. Esta relajación ha dado lugar a un incremento de los riesgos del sistema financiero, que podrían agravarse en 2021, cuando se registre la totalidad del impacto causado por la COVID-19 en las empresas. Las autoridades chinas, conscientes de este peligro, están gestionando selectivamente el aumento de los riesgos financieros del sistema, evitando perjudicar el impacto positivo de las políticas de estímulo para intentar lograr la consolidación $\triangleright$ 
de la recuperación económica iniciada en el año 2020.

Este artículo comienza desgranando la recuperación económica china tras el impacto de la COVID-19 y el papel desempeñado por el sistema bancario. El apartado segundo disecciona la evolución del sistema bancario chino en las últimas décadas para comprender su funcionamiento. El tercer apartado recoge las características propias del sistema financiero chino fruto de su evolución histórica. El cuarto se centra en el papel del sistema financiero chino en la recuperación económica del país tras la epidemia, los riesgos que se están generando y las reacciones de las autoridades. Por último, se concluye señalando el principal riesgo para la recuperación económica china en 2021.

\section{Recuperación de la economía china tras el impacto por la COVID-19}

Mientras el resto del mundo continúa luchando por controlar la pandemia, el 8 de septiembre de 2020 China anunció «la derrota de la COVID-19 en el país» en una ceremonia oficiada por el presidente chino Xi Jinping en Pekín (Yusha y Caiyu, 2020). Este éxito en la gestión del brote de COVID-19 le ha permitido ser la primera economía mundial en iniciar la recuperación económica, convirtiéndose en el único país que alcanzará una tasa positiva de crecimiento en 2020. En concreto, el Fondo Monetario Internacional (IMF, 2020), en sus previsiones macroeconómicas de octubre de 2020, estimó que China será la única economía mundial que evite la recesión en el año 2020 , con un crecimiento del $1,9 \%$ interanual, a diferencia de Estados Unidos $(-4,3 \%)$, la zona euro $(-8,3 \%)$, Japón $(-5,3 \%)$, o la India $(-10,3 \%)$.
Las estrictas medidas de contención de la epidemia aplicadas a partir de febrero, junto con la aprobación de decididas políticas de estímulo económico, permitieron que en el segundo trimestre de 2020 el crecimiento interanual del Producto Interior Bruto (PIB) se situara en el 3,2\%, tras el desplome del $-6,8 \%$ sufrido en los primeros tres meses del año. Las cifras del tercer trimestre confirmaron la recuperación de la economía china, con un crecimiento del $4,9 \%$ trimestral, que permitió retornar a una tasa de crecimiento interanual positiva del 0,7\% (Gráfico 1) (NBSC, 2020).

Esta rápida recuperación de la economía china ha estado impulsada por la inversión y la actividad industrial, mientras que el comportamiento de los hogares y la actividad del sector servicios, que habían sido los dos motores de la economía china en los últimos años, han tenido un comportamiento más dubitativo (Gráfico 2 y Gráfico 3).

La inversión en activos fijos creció un 0,8\% en los primeros nueve meses de 2020 , destacando la recuperación de la inversión inmobiliaria (5,6\% interanual en el tercer trimestre), la inversión pública $(4,1 \%$ interanual en el tercer trimestre) y la inversión en infraestructura digital (33,4\% interanual en el tercer trimestre) (Zenglein et al., 2020; NBSC, 2020).

Por su parte, la actividad industrial creció en el tercer trimestre de 2020 un 6,9\% gracias a la recuperación de la demanda interna y al efecto sustitución de la demanda externa. Este efecto sustitución se debió al colapso causado por la COVID-19 en los sistemas productivos de los países competidores de las exportaciones chinas (i.e., Estados Unidos, Alemania y Japón) que ha provocado que la demanda global se haya dirigido a China, cuyas fábricas se encontraban en condiciones adecuadas para producir desde el mes de abril y, por tanto, $\square$ 


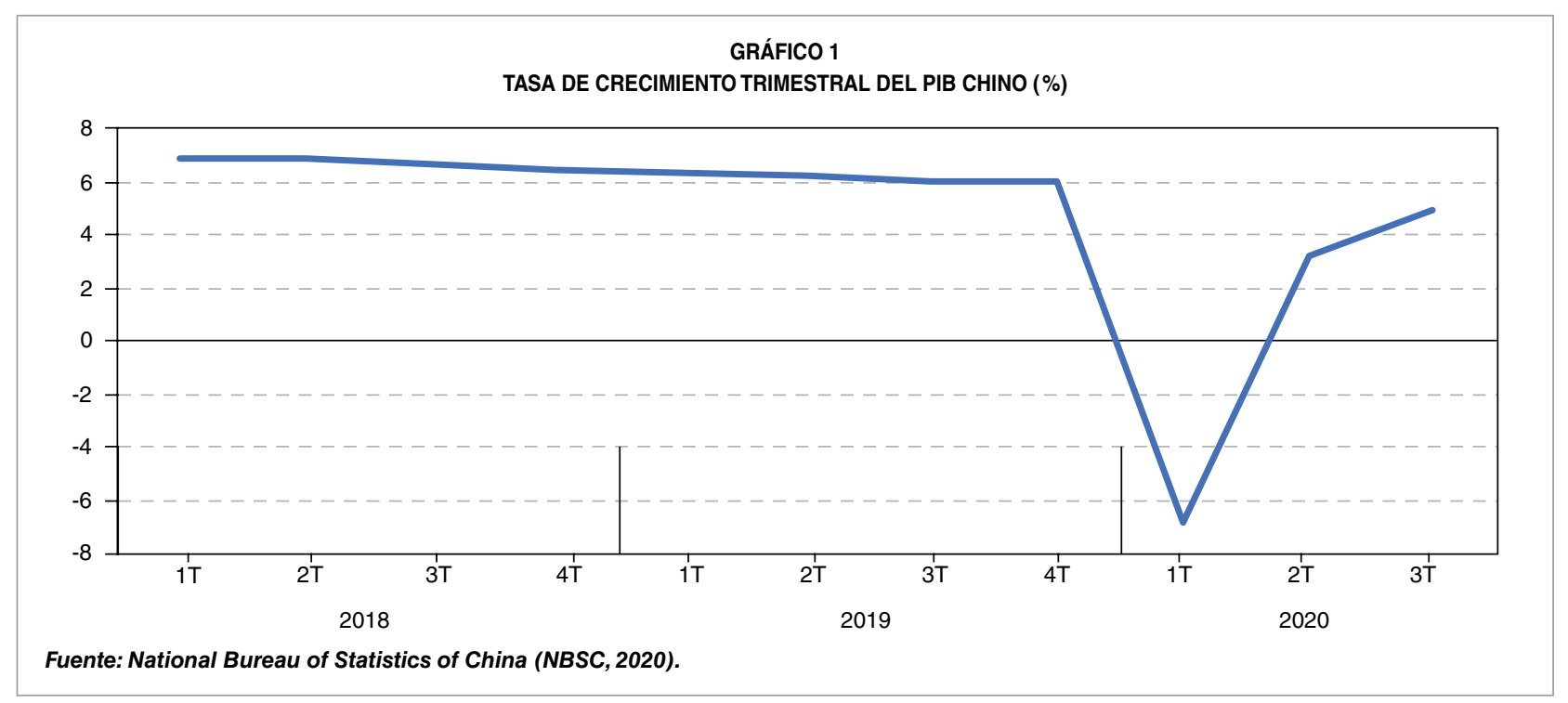

podían satisfacerla. Los sectores más afectados por este fenómeno han sido la electrónica, los productos metálicos, los equipos de transporte y el textil (i.e., material sanitario) (García Herrero y Xu, 2020). Por estos motivos, en el tercer trimestre de 2020 la actividad de las fábricas chinas recuperó los niveles de producción anteriores a la pandemia, como refleja la tasa de utilización de la capacidad industrial, que se situó en el $76,7 \%$, frente al $76,4 \%$ en el mismo periodo de 2019 (NBSC, 2020).

Pese a este buen comportamiento de la economía china, la recuperación presenta síntomas de debilidad y deberá consolidarse en los próximos trimestres. Esto se debe a la persistencia del comportamiento precautorio de

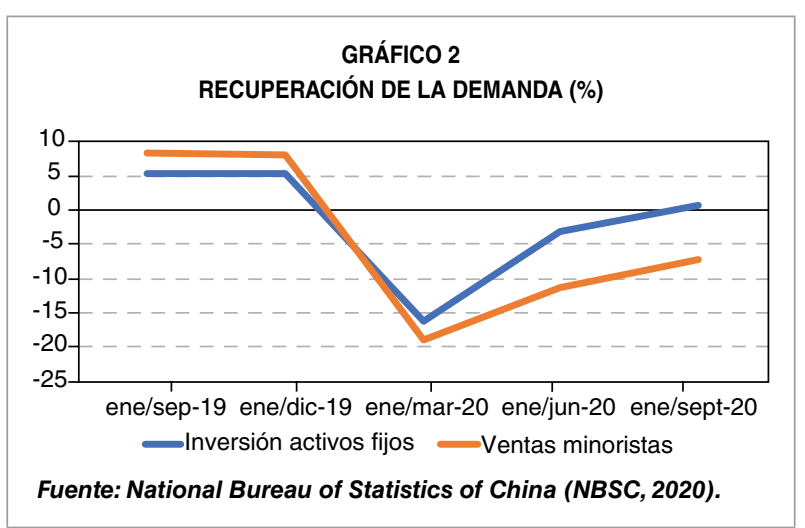

los hogares desde el inicio de la crisis, que se refleja en un descenso de las ventas minoristas del $7,2 \%$ en los nueve primeros meses de 2020 , provocado por la incertidumbre que rodea al mercado laboral ante el temor a posibles rebrotes que, de producirse, afectarían a sectores intensivos en mano de obra como el turismo, la restauración, el transporte o el comercio minorista (Zenglein et al., 2020).

Como se detalla en Tórtola y Alfaraz (2020), la clave de la rápida recuperación de la economía china ha sido la adopción inmediata por parte de las autoridades de políticas expansivas de estímulo fiscal, monetario y sectorial dirigidas a garantizar la liquidez del sistema productivo. En la instrumentalización de gran $\triangleright$

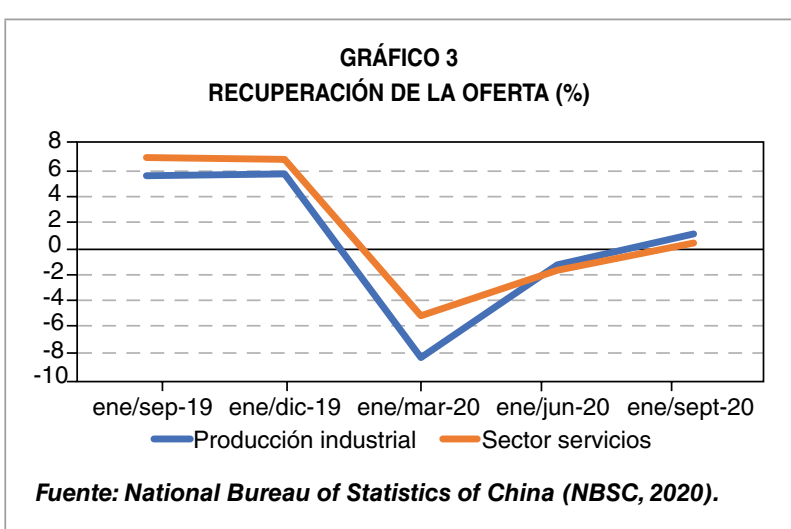


parte de estas políticas expansivas, el sistema bancario ha desempeñado un papel esencial como mecanismo de transmisión. Sin embargo, esta participación de los bancos en la recuperación económica está teniendo un efecto secundario menos deseable, al provocar la reaparición de algunos de los desequilibrios intrínsecos del sistema financiero chino, que las autoridades habían comenzado a corregir en los últimos años (Qian et al., 2020).

\section{Evolución histórica del sistema bancario chino}

En 1978 China inició el proceso de «Reforma Económica y Apertura» que le ha llevado a convertirse en poco más de cuarenta años en la segunda economía mundial, con un 17,9\% del PIB mundial y el $12,1 \%$ del comercio global en el año 2019 (IMF, 2020). Desde el comienzo de este proceso, el sistema bancario ha desempeñado un papel relevante proporcionando la financiación necesaria para lograr los objetivos de desarrollo fijados por las autoridades. Por ese motivo, los bancos chinos han crecido en las últimas décadas en paralelo al desarrollo económico del país hasta convertirse en los bancos más grandes del mundo por volumen de activos (Tabla 1) (Huang y Wang, 2018; Ali, 2020).

\subsection{Origen y crecimiento}

El origen del actual sistema bancario chino se sitúa en 1979, cuando las autoridades chinas aprobaron la creación de tres grandes bancos estatales especializados para conceder financiación a las empresas de los sectores objetivo dentro de la estrategia de desarrollo del Gobierno. Los tres bancos fueron el China Construction Bank (CCB), especializado en el sector de la construcción; el Bank of China (BoC), para operaciones en divisas; $y$ el Agricultural Bank of China (ABC), para el sector agrícola. En 1984, el regulador chino aprobó la creación de un cuarto gran banco estatal, el Industrial \& Commercial Bank of China (ICBC), especializado en financiación urbana comercial. Al mismo tiempo, junto a estos cuatro grandes bancos estatales, en la década de los ochenta surgieron numerosos bancos domésticos a nivel provincial y local. $D$

TABLA 1

RANKING DE BANCOS MÁS GRANDES DEL MUNDO POR VOLUMEN DE ACTIVOS

(Billones de dólares)

\begin{tabular}{|c|c|c|c|c|c|c|c|}
\hline \multicolumn{4}{|c|}{1996} & \multicolumn{4}{|c|}{2020} \\
\hline \multicolumn{2}{|r|}{ Banco } & \multirow{3}{*}{$\begin{array}{l}\text { País } \\
\text { Alemania } . . . \\
\text { Japón }\end{array}$} & \multirow{2}{*}{$\begin{array}{c}\text { Activos } \\
0,503\end{array}$} & \multicolumn{2}{|r|}{ Banco } & \multirow{2}{*}{$\begin{array}{l}\text { País } \\
\text { China }\end{array}$} & \multirow{2}{*}{$\begin{array}{c}\text { Activos } \\
4,676\end{array}$} \\
\hline 1 & Deutsche Bank & & & 1 & ICBC & & \\
\hline 2 & UFJ Bank. & & 0,501 & 2 & China Construction Bank & China $\ldots \ldots \ldots \ldots \ldots \ldots \ldots \ldots$ & 3,905 \\
\hline 3 & Sumitomo Bank . & Japón .. & 0,500 & 3 & Agricultural Bank of China & China & 3,739 \\
\hline 4 & Dai-Ichi Kangyo Bank . & Japón ... & 0,499 & 4 & Bank of China & China & 3,411 \\
\hline 5 & Fuji Bank... & Japón .. & 0,487 & 5 & JPMorgan Chase \& Co. & EE UU & 3,213 \\
\hline 6 & Sakura Bank. & Japón & 0,478 & 6 & Mitsubishi UFJ Financial Group .. & Japón & 3,183 \\
\hline 7 & Bank of Tokyo-Mitsubishi .. & Japón & 0,475 & 7 & BNP Paribas ... & Francia .. & 2,945 \\
\hline 8 & Norinchukin Bank .... & Japón & 0,430 & 8 & HSBC Holdings & Reino Unido & 2,922 \\
\hline 9 & Crédit Agricole... & Francia & 0,386 & 9 & Bank of America .. & EE UU & 2,742 \\
\hline 10 & ICBC & China & 0,374 & 10 & Crédit Agricole Group & Francia ... & 2,470 \\
\hline
\end{tabular}

Fuente: Huang y Wang (2018) y Ali (2020). 
Posteriormente, durante la década de los noventa, las autoridades aprobaron la creación de tres nuevos bancos especializados para reforzar las políticas de apertura económica del Gobierno: Agricultural Development Bank of China (ADBC), China Development Bank (CDB) y Export-Import Bank of China (Chen y Vinson, 2016).

Un cambio muy relevante en el sistema bancario chino se produjo en 1995 con la aprobación de la Commercial Banking Law, por la que los cuatro grandes bancos estatales se convertían en bancos comerciales, responsables de gestionar sus propias operaciones y controlar sus riesgos de acuerdo con la regulación nacional del sistema bancario. Pese a ello, el Gobierno chino continuó desempeñando un papel principal en la actividad bancaria, influyendo para la financiación de las operaciones de las empresas estatales alineadas con los objetivos de los Planes Quinquenales de Desarrollo. Esta operativa y la reducida eficiencia de las empresas públicas provocaron que, a finales de los noventa, el nivel de morosidad de los grandes bancos estatales se situara entre el $30 \%$ y el $40 \%$ (Bonin y Huang, 2001).

\subsection{Reforma y liberalización del sistema bancario}

Esta elevada tasa de morosidad obligó a las autoridades a iniciar un proceso de reforma del sistema bancario, para mejorar su solvencia, que se ejecutó en cuatro etapas. La primera, en 1998, involucró a los cuatro grandes bancos sistémicos. En la segunda, en 2003, se centraron en el China Construction Bank y en el Bank of China. La tercera, en 2005, en el ICBC. Y la última, en 2008, en la entidad sistémica más débil, el Agricultural Bank of China. En cada etapa se realizaron inyecciones de capital con financiación pública y limpieza del balance de préstamos impagados a través de su provisión contra el capital preexistente o su transferencia a sociedades de gestión de activos (AMCs) (García-Herrero y Santabárbara, 2009).

En total, entre las cuatro fases, se inyectaron en el sistema bancario chino aproximadamente 100.000 millones de dólares (a precios constantes de 2008) en forma de capital y se eliminaron aproximadamente 530.000 millones de dólares en préstamos impagados. A su vez, el proceso de reestructuración bancaria estuvo acompañado por la aprobación de nuevas regulaciones para liberalizar el sistema, aumentando la competencia y reduciendo las restricciones a los flujos de capital transfronterizo (García-Herrero y Santabárbara, 2013).

\subsection{Crisis financiera global (2008)}

Ante el estallido de la crisis financiera global en 2008, las autoridades chinas recortaron los tipos de interés en 108 puntos básicos, el mayor recorte desde los años noventa, y aprobaron un agresivo paquete de estímulo fiscal por valor de 588.000 millones de dólares (cerca del $13 \%$ del PIB chino en 2008). Este plan de estímulo fiscal incluyó un ambicioso plan de inversión en infraestructuras y vivienda pública que permitió fijar, como objetivo de crecimiento, el $8 \%$ del PIB en 2009. La principal novedad de este plan fue su canalización a través de la financiación bancaria a los proyectos de inversión de los Gobiernos locales, en lugar de mediante gasto público directo del Gobierno central. De esta manera, en 2009 y 2010 la financiación bancaria alcanzó los 2,8 billones de dólares (equivalentes al $56 \%$ de la economía china en 2009), que fue dirigida mayoritariamente a las empresas estatales a nivel $\triangleright$ 
de ciudad, municipio y provincias. En este contexto surgieron los Vehículos de Financiación del Gobierno Local (LGFV, por sus siglas en inglés), que consistían en entidades respaldadas por los Gobiernos locales para recaudar fondos destinados al desarrollo de proyectos inmobiliarios y de infraestructura «fuera de balance", lo que eliminaba en la práctica el techo de gasto fiscal de los Gobiernos locales.

En 2011, pese a la aparición de tensiones inflacionistas y que la economía china había superado los efectos de la crisis financiera, el sistema bancario continuó su expansión, impulsando un crecimiento económico basado en la inversión financiada mediante crédito bancario, dando lugar a un incremento de los activos bancarios de 20,7 billones de dólares entre 2012 y 2017.

Sin embargo, debido a que la mayor parte de la financiación estaba destinada a las grandes empresas estatales, durante esos años comenzaron a proliferar nuevos productos e instituciones financieras no bancarias que ofrecían financiación al resto de agentes económicos en unas condiciones menos estrictas y más arriesgadas que la banca formal gracias a la débil supervisión regulatoria. Estas instituciones, conocidas como "banca en la sombra", se integraron en el sistema financiero, complementando al crédito bancario tradicional y dando lugar a un sistema con mayor diversidad de fuentes de financiación, pero más inestable.

Gracias a esta expansión del crédito bancario tradicional y a la proliferación de la banca en la sombra, a finales de 2017 China se había convertido en el mayor sistema bancario del mundo por volumen de activos, cuadruplicando su nivel de 2008 (Wright y Rosen, 2018; CBIRC, 2020) (Gráfico 4).

La magnitud de esta evolución queda reflejada tanto por el liderazgo mundial de los bancos estatales chinos por volumen de activos como por su capacidad de innovación, lo que le ha permitido ser la economía con mayor nivel de penetración de los pagos digitales a través de plataformas como Alipay y Wechat Payments (Liang, 11 de julio de 2020) y desarrollar el yuan digital (Devonshire-Ellis y Qong, 20 de agosto de 2020).

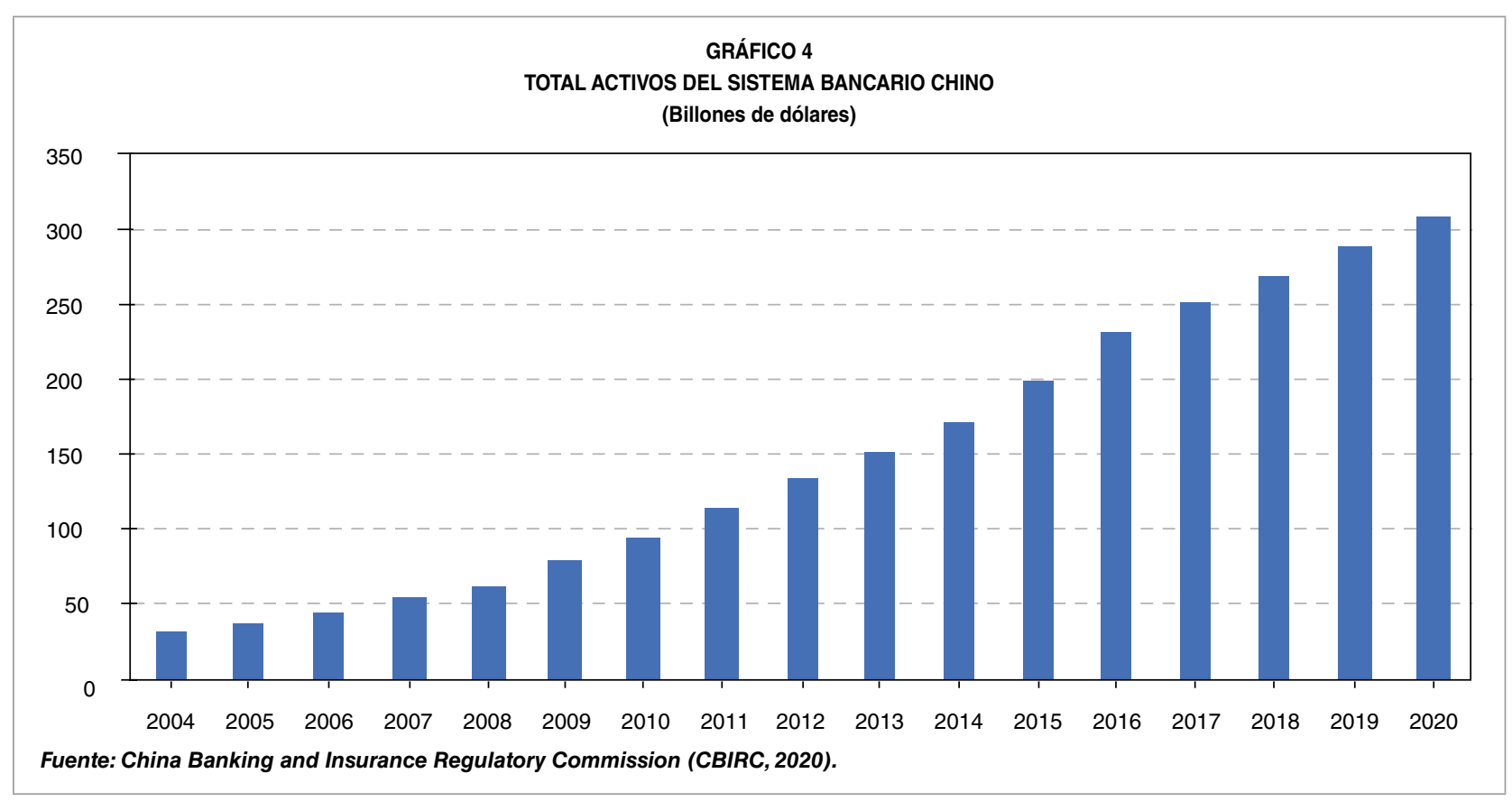




\section{Características intrínsecas del sistema financiero chino}

La evolución del sistema financiero chino, durante las últimas décadas, se ha basado en unas características particulares que lo convierten en un sistema único en el mundo. En primer lugar, está dominado por la actividad bancaria, que constituye el principal canal de financiación de hogares y empresas. Sin embargo, siguiendo las indicaciones de las autoridades chinas, los bancos muestran una clara preferencia por los prestatarios estatales frente al sector privado, ya que cuentan con el respaldo de garantías gubernamentales, lo que no contribuye a lograr una asignación eficiente de los recursos basada en la rentabilidad de las operaciones. En segundo lugar, las operaciones de compra o venta de bonos se ejecutan en un mercado poco profundo por el dominio de las empresas estatales en el mercado de bonos corporativos. En tercer lugar, el mercado de valores resulta poco fiable, ya que prima la actividad especulativa como consecuencia de la falta de información significativa y de una regulación que facilite las decisiones de inversión basadas en las condiciones de las empresas. Por último, es un sector estrictamente controlado por el Gobierno, lo que explica el posicionamiento de las entidades estatales en el mercado y la tímida apertura al capital extranjero (Wright y Rosen, 2018).

Estas características intrínsecas del sistema financiero chino y el gran crecimiento experimentado a partir de la crisis financiera global han dado lugar a la aparición de algunos desequilibrios estructurales exclusivos del sistema chino:

- Dualidad del mercado bancario. A pesar de su ineficiencia productiva frente al sector privado, las empresas estatales tienen más fácil acceso al crédito bancario por sus garantías gubernamentales, convirtiéndose en clientes preferenciales de los grandes bancos estatales comerciales, mientras que las pymes y los Gobiernos locales se financian mayoritariamente a través de los bancos provinciales de menor tamaño. Esto implica que los bancos de menor tamaño, que cuentan con menor capitalización, asumen un mayor riesgo de insolvencia al carecer de las garantías gubernamentales en sus operaciones con las empresas privadas y al contar entre sus principales clientes con los Gobiernos locales (a través de los LGFV), que se encuentran altamente endeudados (Cong et al., 2019).

- Riesgo moral generalizado. La cobertura de la financiación a empresas estatales con garantías gubernamentales implica que, en la práctica, estos préstamos no conllevan (casi) riesgos, lo que incentiva el crecimiento de estos préstamos bajo la consideración de que el Gobierno no permitirá su quiebra en ningún caso. Esta situación genera, a su vez, un incremento en el coste de financiación para las empresas privadas para compensar la carencia de este tipo de garantías, dando lugar a una asignación ineficiente de los recursos financieros (Wright y Rosen, 2018).

- Banca en la sombra. Debido al plan de estímulo fiscal de 2008, a las dificultades de las empresas privadas para financiarse y a la falta de regulación específica, a partir de 2008 comenzaron a proliferar productos crediticios sin supervisión prudencial. Estos productos son ofrecidos por instituciones bancarias (fuera de balance) y no bancarias, y pueden cubrir desde operaciones de financiación de activos patrimoniales hasta préstamos $\triangleright$ 
al consumo a corto plazo. Este sistema de financiación paralelo es de difícil control para las autoridades y acumula un elevado riesgo por el reducido nivel de garantías exigidas y el alto grado de apalancamiento de las operaciones. En 2017, la banca en la sombra alcanzó los 15,4 billones de dólares, con crecimientos anuales superiores al 20\% desde 2008 (Allen y Gu, 2020; CBIRC, 2020).

A partir de 2016, debido al riesgo sistémico generado por el gran tamaño de la banca en la sombra, las autoridades chinas aprobaron nuevos paquetes regulatorios para reducir el apalancamiento financiero de estas operaciones, aumentar su transparencia y mejorar la gestión de riesgos y control de estas prácticas (Sutton y Taylor, 2020). Como consecuencia de estas medidas, el stock de operaciones «en la sombra» en China se redujo en 2019 hasta los 3 billones de dólares (CBIRC, 2020).

Sin embargo, el estallido de la pandemia de la COVID-19, en enero de 2020, y la decisión de las autoridades chinas de recurrir de nuevo al sistema bancario como principal instrumento para canalizar sus políticas de estímulo han obligado a una relajación del control de riesgos e incremento del crédito que está provocando un aumento de los desequilibrios del sistema financiero chino.

\section{El sector financiero chino tras la COVID-19}

Desde el inicio de la epidemia, las autoridades chinas han aprobado numerosas medidas de estímulo para intentar contrarrestar los efectos negativos de la COVID-19 sobre la actividad económica. Estas políticas de estímulo fiscal y monetario se han caracterizado por un mayor enfoque sectorial que las medidas ejecutadas durante la crisis financiera de 2008. Al igual que en crisis anteriores, sin embargo, las autoridades han recurrido también al sistema bancario como mecanismo principal de transmisión de sus políticas e incluso han reforzado su participación. Para ello, en diciembre de 2020, el Ministerio de Finanzas chino revisó el Sistema de Evaluación del Desempeño de la Banca Comercial para incluir como parte de la evaluación, desde el 1 de enero de 2021, la contribución de cada banco al desarrollo nacional y economía real, la calidad de este desarrollo, el control y la prevención de riesgos, y la eficiencia operativa (Ministerio de Finanzas, 2020).

Por el lado de la política monetaria, la estrategia del Banco Central de China (PBoC) se centró en tres objetivos. En primer lugar, incrementar el nivel de liquidez en el sistema con tres recortes en el coeficiente legal de caja de los bancos. En segundo lugar, suavizar las condiciones para la concesión de crédito y abaratar los préstamos a las empresas más afectadas por la epidemia. La tasa de los préstamos a medio plazo (un año) para instituciones financieras pasó del 3,25\% a principios de año al 2,95\% en mayo, mientras que la tasa preferencial de préstamos a un año se redujo en abril en 30 puntos básicos hasta situarse en el 3,85\%, donde se ha mantenido desde entonces. Por último, se buscó aliviar los problemas de liquidez de las pymes mediante recomendaciones a las instituciones financieras sobre la adopción de soluciones de crédito innovadoras, la optimización de los procedimientos de aprobación de préstamos y un aumento del $40 \%$ en los préstamos concedidos por los bancos comerciales a pymes y microempresas. En total, se estima que el apoyo a través de los bancos a las $D$ 
empresas ascendió a 230.000 millones de dólares en 2020 (aproximadamente el $75 \%$ de sus beneficios de 2019) (Tórtola y Alfaraz, 2020; Keqiang, 2020; Cerqueira y Huang, 2020).

Por el lado de la política fiscal, además de aprobar medidas para la estabilización del empleo y la reducción temporal de cargas a las empresas, con especial atención a las pymes (i.e., reducción de impuestos o exenciones en la seguridad social). Las autoridades han apostado por la emisión de «bonos de propósito especial» a nivel provincial para financiar proyectos de infraestructuras, urbanismo y energía por valor de $\mathbf{5 2 5 . 0 0 0 ~ m i l l o n e s ~ d e ~ d o ́ l a r e s , ~ c o n - ~}$ virtiéndose los bancos en los principales compradores de estos bonos «de propósito especial» emitidos por los Gobiernos locales a través de los LGFV (Keqiang, 2020).

Para que las entidades bancarias pudieran cumplir con su función dentro de estas políticas de estímulo del Gobierno, las autoridades se han visto obligadas a suavizar la regulación financiera para que los bancos pudieran incrementar sus balances, con independencia de si empeoraba o no su solidez financiera en el futuro (García Herrero y Ng, 2020a). Debido a esta relajación de los controles financieros, algunos de los problemas estructurales del sector financiero chino han comenzado a agudizarse durante el segundo semestre de 2020, lo que ha obligado a las autoridades chinas a intervenir de manera selectiva para intentar controlarlos sin reducir el impulso expansivo de las políticas aprobadas.

\subsection{Calidad de los activos}

En líneas generales, los bancos pequeños y medianos (urbanos y rurales) prestan en mayor proporción a las pymes $(70 \%)$ en comparación con los grandes bancos comerciales estatales, cuya cartera de clientes se compone principalmente de las grandes empresas estatales.

Las recomendaciones del Banco Central de China, a todos los bancos, es facilitar la financiación a pymes y microempresas, tendencia que se ha mantenido durante 2020 aunque los bancos medianos y pequeños, además de prestar más a las pymes y al sector privado, han destinado un tercio de sus préstamos a sectores muy afectados por la pandemia, como manufacturas (al por mayor y al por menor), transporte, almacenamiento y servicios postales. Esta dinámica está provocando el deterioro de la calidad de los activos de los bancos pequeños y medianos, lo que se traduce en un mayor riesgo de insolvencia para estas entidades (Liang, 11 de julio de 2020).

Hasta el momento, la morosidad del sistema se ha incrementado moderadamente desde el 1,86\% del último trimestre de 2019 hasta el $1,96 \%$ del tercer trimestre de 2020. Sin embargo, se prevé que en 2021 continúe creciendo debido a que la evolución económica negativa de 2020 impactará en su totalidad en las empresas en 2021, afectando especialmente a la calidad de los activos de los bancos locales y provinciales. Por este motivo, los bancos han comenzado a tomar medidas, reestructurando préstamos dudosos por valor de 261.000 millones de dólares (aumento del $25 \%$ respecto a 2019 ) y cancelando préstamos por valor de 118.500 millones de dólares en los primeros nueve meses de 2020 (García Herrero y Ng, 2020b).

\subsection{Rentabilidad de los activos}

Los intereses representan más del $75 \%$ de los ingresos de los bancos chinos. Por ello, $\square$ 
el recorte en 30 puntos básicos de la tasa preferencial de préstamos a un año durante 2020 ha afectado negativamente el margen de interés neto de las entidades bancarias (PBoC, 20 de abril de 2020) (Liang, 11 de julio de 2020). Esta reducción de la rentabilidad por intereses ha sido parcialmente compensada por el aumento del volumen de préstamos del 13,5\% interanual en el tercer trimestre de 2020, pese a lo cual, los bancos están intentando diversificar sus fuentes de ingresos mediante comisiones por servicios de compensación y liquidación, consultoría o servicios de agencia. El objetivo es evitar que la reducción de ingresos por intereses, que ya ha generado una caída del 8,3\% interanual en sus beneficios hasta el tercer trimestre de 2020, ponga en peligro la propia viabilidad de los bancos (CBIRC, 2020; Cheng, 2020; García Herrero y Ng, 2020a).

Por su parte, el Banco Central de China y la Comisión Reguladora de Banca y Seguros de China (CBIRC) están preparando una regulación específica para los bancos considerados «demasiado grandes para quebrar» con el fin de fortalecer su capacidad de prevención y absorción de riesgos y mejorar su rentabilidad. En la lista inicial de bancos que estarán sujetos a esta regulación están incluidos los cuatro grandes bancos estatales y otros veinticinco bancos chinos calificados como entidades de importancia sistémica (Chen, 5 de diciembre de 2020).

\subsection{Impago de bonos corporativos}

Como alternativa a los préstamos bancarios, las empresas (privadas y públicas) han recurrido en los últimos años a la emisión de bonos corporativos para financiarse a menor coste. En 2020, el mercado de bonos corporativos doméstico de China alcanzó un volumen de 15 billones de dólares, convirtiéndose en el segundo mercado más grande del mundo (Wang, 24 de noviembre de 2020).

Sin embargo, pese a la rápida recuperación de la economía china tras el impacto provocado por la COVID-19, la capacidad de las empresas para pagar el servicio de su deuda se ha debilitado y, desde el mes de noviembre de 2020, han comenzado a sucederse impagos de bonos corporativos. Esto se debe a la caída de los precios industriales, que se mantienen en tasas negativas desde el inicio de la pandemia, lo que ha reducido los beneficios industriales de las empresas estatales un $7,5 \%$ durante los diez primeros meses de 2020 (NBSC, 2020). A esta caída de los beneficios se le añade el compromiso de las empresas estatales con el Gobierno central para mantener el empleo y el nivel retributivo de sus empleados (MERICS, 2020; EIU, 4 de noviembre de 2020). Algunos ejemplos de impagos ocurridos entre noviembre y diciembre de 2020 son: Huachen Automotive Group Holdings, propiedad del Gobierno provincial de Liaoning, que ha entrado en bancarrota tras no poder hacer frente al pago de un bono por valor de 152 millones de dólares y que posee una deuda de 5.200 millones de dólares; Yongcheng Coal and Electricity, con sede en la provincia de Henan, con un impago de 461 millones de dólares; o Tsinghua Unigroup, empresa de semiconductores filial de la universidad pública de Tsinghua, que no ha podido hacer frente a pagos por valor de 2.600 millones de dólares.

Debido a esta oleada de impagos, a mediados de noviembre, el Banco Central de China se vio obligado a inyectar 122.000 millones de dólares para mantener los mercados financieros en funcionamiento, y ya ha anunciado que los inversores deberán asumir los riesgos $\triangleright$ 
de sus propias inversiones, lo que dejaría sin efecto las garantías gubernamentales que soportan a las empresas estatales con el objetivo de introducir cierta disciplina en el mercado (MERICS, 2020).

\subsection{Banca en la sombra}

La reducción de los tipos de interés y el retraso de la entrada en vigor de la nueva regulación para la gestión de activos hasta 2021 han dado lugar a un moderado aumento de la banca en la sombra que se estima en 100.000 millones de dólares durante la primera mitad de 2020 (Cerqueira y Huang, 2020; García Herrero y Ng, 2020a). Pese a ello, las autoridades chinas han confirmado que continuarán regulando para reducir este tipo de financiación y velando por mantener la estabilidad del sistema financiero frente a la innovación financiera. Prueba de ello es que, en agosto de 2020, la Corte Suprema de China decidió reducir las tasas de interés sobre los préstamos informales, disminuyendo la rentabilidad de estas operaciones (Taylor y Li, 2020).

Además, en el último trimestre de 2020, las autoridades están ejerciendo un mayor control sobre la industria fintech, que ya es considerada parte del sistema bancario «en la sombra» al ofrecer productos de préstamo y ahorro similares a los del sector bancario tradicional, pero sin estar sujetos a las mismas regulaciones (i.e., coeficiente de caja o requisitos de provisiones) (Liang, 11 de julio de 2020). Como resultado de este mayor control, las autoridades chinas decidieron suspender la Oferta Pública Inicial (OPI) de Ant Group, destinada a convertirse en la mayor OPI de la historia, a solo 36 horas de su salida a bolsa (EIU, 17 de noviembre de 2020), y que posteriormente vino acompañada de la publicación de estrictas reglas para prevenir los riesgos sistémicos que plantean las actividades de las grandes fintech (Guo, 8 de diciembre de 2020).

\section{Conclusiones}

El sistema bancario ha desempeñado un papel central en el desarrollo de la economía china desde el inicio del proceso de reforma y apertura del país a finales de los años setenta. Al mismo tiempo, ha constituido el principal mecanismo de transmisión utilizado por las autoridades para ejecutar las políticas de estímulo aprobadas durante las crisis económicas acontecidas en las últimas décadas.

La rápida recuperación económica lograda por China tras la crisis de la COVID-19 se ha debido a las estrictas medidas de contención que permitieron eliminar la epidemia dentro de China en muy poco tiempo y a un ambicioso plan de estímulo que se ha vuelto a canalizar, en gran medida, a través del sistema bancario. Sin embargo, esta intervención de los bancos ha acentuado los riesgos del sistema al aumentar el volumen de los activos bancarios en un $13,5 \%$ interanual en el tercer trimestre de 2020, mientras se deterioraban los balances de las empresas chinas por el efecto de la pandemia.

Por tanto, el principal riesgo que afronta el sistema financiero chino, que podría poner en peligro la recuperación económica del país, es el posible incremento de los impagos de bonos corporativos y préstamos bancarios y no bancarios empresariales, como ha ocurrido en los meses de noviembre y diciembre de 2020, cuyo impacto podría acentuarse tras el inesperado anuncio de las autoridades chinas de no acudir al rescate de estos inversores. Este $\triangleright$ 
anuncio de las autoridades pretende reducir el riesgo moral del sistema financiero chino, aunque afecta a uno de los pilares fundamentales del sistema, que era la confianza en que las autoridades chinas (a nivel local o central) acudirían al rescate de las empresas y entidades públicas en crisis.

Por todo ello, el primer semestre de 2021 será determinante para comprobar la estabilidad del sistema financiero chino y el impacto de esta nueva política de las autoridades chinas para gestionar los problemas financieros de las empresas de forma más acorde con las reglas del mercado.

\section{Bibliografía}

Ali, Z. (7 de abril de 2020). The world's 100 largest banks, 2020. S\&P Global Market Intelligence. https://www.spglobal.com/marketintelligence/en/ news-insights/research/the-worlds-100-largestbanks-2020

Allen, F., y Gu, X. (2020). Shadow Banking in China compared to other countries. The Manchester School. Willey. Special Issue Article. https://doi. org/10.1111/manc.12331

Bonin, J., y Huang, Y. (2001). Dealing with the bad loans of the Chinese banks. Journal of Asian Economics, 12(2), 197-214. https://doi. org/10.1016/S1049-0078(01)00082-3

CBIRC, China Banking and Insurance regulatory Commission (2020). Statistics (varias). Pekín. China. https://www.cbirc.gov.cn/en/

Cerqueira, O., y Huang, B. (2020). China Banking Monitor 2020. BBVA Research.

Chen, J. (5 de diciembre de 2020). China unveils standards for 'too big to fail'. China Daily. https:// www.chinadaily.com.cn/a/202012/05/WS5fcb2013a31024ad0ba99f31.html

Chen, L., y Vinson, S. (2016). An Overview of the Chinese Banking System: Its History, Challenges and Risks. Journal of Business and Economics,
7 (10), 1613-1617. http://www.academicstar.us/ issueshow.asp?daid $=1926$

Cheng, E. (15 de octubre de 2020). China is open to taking on more debt if that's it takes to support the economy. CNBC. https://www.cnbc.com/ 2020/10/16/china-is-open-to-more-debt-to-support-its-economy.html? \&qsearchterm= China\%20is\%20open\%20to\%20taking\%20 on $\% 20$ more $\% 20$ debt $\% 20$ if $\% 20$ that $\%$ E2\%80\%99s\%20what\%20it\%20takes\%20 to\%20support\%20the\%20economy

Chinese regulators suspend Ant Group (4 de noviembre de 2020). The Economist Intelligence Unit (EIU).

Cong, L., Gao, H., Ponticelli, J., y Yang, X. (2019). Credit Allocation Under Economic Stimulus: Evidence from China. The Review of Financial Studies, 32(9), 3412-3460. https://doi.org/10.1093/ rfs/hhz008

Devonshire-Ellis, C., y Wong, D. (20 de agosto de 2020). When Can I Buy, Use, and Trade China's Digital Yuan? China Briefing. https://www. china-briefing.com/news/when-can-i-buy-useand-trade-chinas-digital-yuan/

García Herrero, A., y Ng, G. (2020a). Catch-22 for Chinese Banks: How to support growth and keep financial soundness simultaneously? Natixis.

García Herrero, A., y Ng, G. (2020b). Chinese Banks show the first signs of recovery, but challenges remain on liquidity, asset quality and even solvency. Natixis.

García-Herrero, A., y Santabárbara, D. (2009). Una valoración de la reforma del sistema bancario de China. Boletín Económico de ICE (2972). http:// www.revistasice.com/index.php/BICE/article/ view/4787

García-Herrero A., y Santabárbara D. (2013). An Assessment of China's Banking System Reform. En Kaji S., Ogawa E. (eds), Who Will Provide the Next Financial Model? Springer, Tokio. https:// doi.org/10.1007/978-4-431-54282-7_15

García-Herrero, A., y Xu, J. (2020). China Bound to Increase Global Export Share Again. Natixis. $\triangleright$ 
Guo, Y. (8 de diciembre de 2020). Fintech Giants Could Pose «New Systemic Risks», Warns Banking Watchdog Chief. Caixin.

Huang, Y., y Wang, X. (2018). Strong on quantity, weak on quality: China's financial reform between 1978 and 2018. En R. Garnaut, L. Song, \& C. Fnag (Eds.), China's 40 years of reform and development: 1978-2018 (291-312). ANU Press, The Australian National University. https://press. anu.edu.au/publications/series/china-update/ china \%E2 \%80\%99s-40-years-reform-anddevelopment-1978\%E2\%80\%932018

IMF, The International Monetary Fund (2020). World Economic Outlook, October 2020: A Long and Difficult Ascent. https://www.imf.org/en/Publications/WEO/Issues/2020/09/30/world-economicoutlook-october-2020

Keqiang, L. (2020). Informe sobre la Labor del Gobierno. III Sesión de la XIII Asamblea Popular Nacional. Observatorio de la Política China. https://politica-china.org/secciones/informe-sobrela-labor-del-gobierno-presentado-el-22-demayo-de-2020-ante-la-iii-sesion-de-la-xiiiasamblea-popular-nacional

Liang, Y. (11 de julio de 2020). China's Banking Sector Faces Multiple Challenges Alter COVID-19. The Diplomat.

MERICS (The Mercator Institute for China Studies) (2020). Bond defaults: Beijing weeds out state-owned enterprises. https://merics.org/en/ short-analysis/bond-defaults-beijing-weedsout-state-owned-enterprises

Ministerio de Finanzas (15 de diciembre de 2020). Aviso sobre la adopción de «Medidas de Evaluación del Desempeño de la Banca Comercial». Circular 124. Pekín. China.

NBSC (National Bureau of Statistics of China) (2020). Press Release (varias). http://www.stats. gov.cn/english/

PBoC (Banco Central de la R.P. de China) (20 de abril de 2020). Announcement on Loan Prime Rate.
Qian, H., Ekberg, J., Yip, J., Chen, J., He, L., y He, L. (2 de abril de 2020). Covid-19 and financial services in China. Challenges and Opportunities for the Chinese Financial Services Sector. Oliver Wyman. https://www.oliverwyman.com/our-expertise/insights/2020/apr/chinese-finance-and-covid-19.html

SOE defaults rattle China's corporate bond market (17 de noviembre de 2020). The Economist Intelligence Unit (EIU).

Sutton, M., y Taylor, G. (2020). Shadow Financieng in China. Bulletin-December 2020. Reserve Bank of Australia. https://www.rba.gov.au/publications/bulletin/2020/dec/shadow-financing-in-china.html

Taylor, M., y Li, L. (2020). China's shadow banking sector dims as formal bank lending dominates new credit supply. Moody's Investors Service. https://www.moodys.com/research/MoodysChinas-shadow-banking-sector-dims-as-formalbank-lending--PBC_1247149

Tórtola, C., y Alfaraz, N. (2020). El impacto de la COVID-19 en la economía china. Boletín Económico de ICE (3125). https://doi.org/10.32796/ bice.2020.3125.7041

Wang, Y. (24 de noviembre de 2020). State Council poised to dial up regulation of corporate defaults. Global Times. https://www.globaltimes.cn/content/1207859.shtml

Wright, L., y Rosen, D. (2018). Credit and credibility. Risks to China's economic resilience. Center for Strategic \& International Studies (CSIS). https:// rhg.com/wp-content/uploads/2018/10/181003_ CreditandCredibility_final.pdf

Yusha, Z., y Caiyu, L. (8 de septiembre de 2020). Xi hails unity of China's $1.4 \mathrm{~b}$ people in COVID-29 battle. Global Times. https://www.globaltimes.cn/ content/1200304.shtml

Zenglein, M., Kärnfelt, M., y Chimits, F. 2020). China's economic recovery picks up pace. The Mercator Institute for China Studies (MERICs). https://merics.org/en/tracker/chinas-economicrecovery-picks-pace 
